\title{
Cholinergic Deafferentation of the Neocortex Using 192 IgG-Saporin Impairs Feature Binding In Rats
}

\author{
Leigh C. P. Botly ${ }^{1}$ and Eve De Rosa ${ }^{1,2}$ \\ ${ }^{1}$ Department of Psychology, University of Toronto, Toronto, Ontario, Canada M5S 3G3, and 2Rotman Research Institute, Toronto, Ontario, Canada M6A 2E1
}

The binding problem refers to the fundamental challenge of the CNS to integrate sensory information registered by distinct brain regions to form a unified neural representation of a stimulus. Although the human cognitive literature has established that attentional processes in frontoparietal cortices support feature binding, the neurochemical and specific downstream neuroanatomical contributions to feature binding remain unknown. Using systemic pharmacology in rats, it has been shown that the neuromodulator acetylcholine is essential for feature binding at encoding, but the neural source of such critical cholinergic neurotransmission has yet to be identified. Cholinergic efferents from the nucleus basalis magnocellularis (NBM) of the basal forebrain provide the majority of the cholinergic input to the neocortex. Accordingly, it was hypothesized that the NBM is the neural source that provides the critical neuromodulatory support for feature binding. To test this hypothesis, rats received bilateral 192 IgG-saporin lesions of the NBM, and their feature binding performance was tested using a forced-choice digging paradigm. Relative to sham-lesioned rats, NBM-lesioned rats were significantly impaired at acquiring a crossmodal feature conjunction (FC) stimulus set that required feature binding, whereas their ability to retrieve an FC stimulus set and to acquire two crossmodal feature singleton stimulus sets, one of greater difficulty than the other but neither requiring feature binding, remained intact. These behavioral findings, along with histological analyses demonstrating positive relationships between feature-binding acquisition and markers of cholinergic activity in frontoparietal regions, reveal the importance of neocortical cholinergic input from the NBM to feature binding at encoding.

\section{Introduction}

We perceive objects as unified wholes, but the mammalian brain is organized such that distinct neural regions are primarily responsible for detecting and processing the different features of an object, such as its shape or color. The unknown mechanism by which unified neural representations of objects are formed is referred to as the binding problem (Treisman and Gelade, 1980). Although the human cognitive literature has established that attentional processes in frontoparietal cortices support feature binding (Cohen and Rafal, 1991; Corbetta et al., 1995; FriedmanHill et al., 1995; Robertson, 2003; Esterman et al., 2007), the neurochemical and specific downstream neuroanatomical contributions to feature binding remain unknown. It was hypothesized that acetylcholine (ACh) would be critical to feature binding given this neuromodulator's presumed role in modulating attention (Sarter et al., 2005) and the well established importance of attention to feature binding (Friedman-Hill et al., 1995; Foster et al., 1999; Tales et al., 2002).

\footnotetext{
Received Feb. 8, 2009; accepted Feb. 24, 2009.

This work was supported by grants from the Natural Sciences and Engineering Research Council of Canada (NSERC), Connaught, and Canada Foundation for Innovation/Ontario Innovation Trust awarded to E.D.R., as well as a postgraduate scholarship from NSERC awarded to L.C.P.B. We thank Maryam Saheb-Al-Zamani, I-Zen Wang, Steve Greening, Mavis Kusi, and Isabel Chiu for help with animal testing.

Correspondence should be addressed to either Leigh C. P. Botly or Eve De Rosa, Department of Psychology, University of Toronto, 100 St. George Street, Toronto, Ontario, Canada M5S3G3. E-mail: leigh@psych.utoronto.ca or derosa@psych.utoronto.ca.

DOI:10.1523/JNEUROSCI.0654-09.2009

Copyright $\odot 2009$ Society for Neuroscience $\quad$ 0270-6474/09/294120-11\$15.00/0
}

In support of this hypothesis, previous data have shown that systemic muscarinic cholinergic blockade with scopolamine impaired the ability of rats to acquire feature conjunction (FC) stimuli that require feature binding, leaving their ability to acquire feature singleton (FS) stimuli that do not require binding intact (Botly and De Rosa, 2007). Moreover, such muscarinic blockade selectively impaired feature binding at encoding, leaving the retrieval of previously learned FC stimuli intact. Such an encoding-retrieval dissociation is consistent with a well supported model of cholinergic function (Hasselmo and McGaughy, 2004).

Based on these pharmacological findings, it was hypothesized that ACh provides the neurochemical support for the attentional processes critical for feature binding. Evidence for this proposition has come from a recent cross-species study using an intramodal version of this paradigm that showed that dividing the attention of humans with a concurrent task yielded a similar pattern of impairment in feature binding to muscarinic blockade in rats, impairing the acquisition of an intramodal FC task while leaving FC retrieval and FS acquisition intact (Botly and De Rosa, 2008).

Although these data implicate ACh in feature binding at encoding, the neural source of such critical cholinergic neurotransmission has yet to be identified. Thus, we hypothesized that the nucleus basalis magnocellularis (NBM) of the basal forebrain (BF) would be critical for feature binding given that its cholinergic efferents provide $90 \%$ of the cholinergic input to the neocortex (Mesulam et al., 1983), including frontoparietal regions implicated in attentional processing (Donner et al., 2002; Behrmann et al., 2004; Bucci and Chess, 2005; Constantinidis, 2006; Bucci, 2009). 


\section{A. Trial Type}

Target ( $\mathrm{T})$

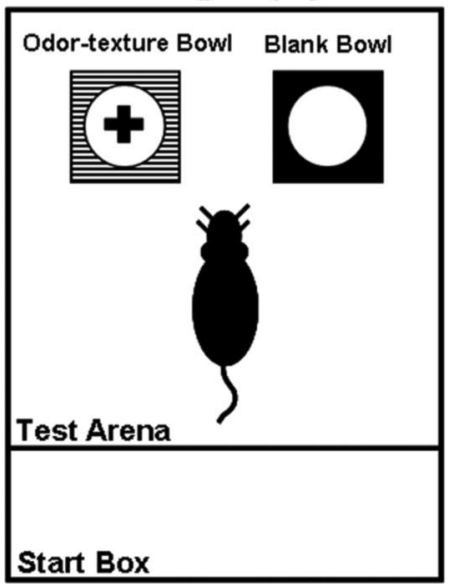

Distractor (D)

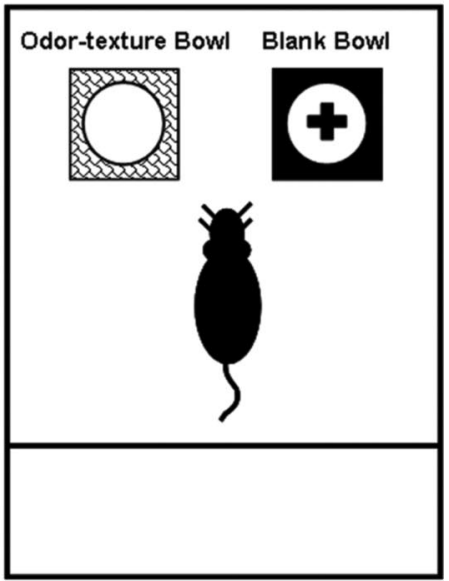

\section{B. Session}

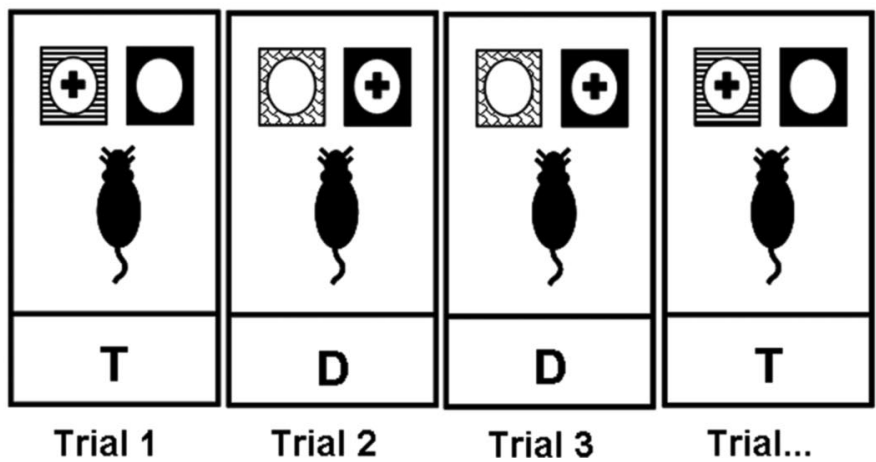

Figure 1. A, Illustration of the two different trial types, target and distractor, of the forced-choice digging tasks. On target trials, the rewarded $(+)$ stimulus was the odor-texture bowl, whereas on distractor trials, the rewarded stimulus was the blank bowl. $\boldsymbol{B}$, Illustration of a typical session. On every trial, rats were simultaneously presented with two digging bowls: an odortexture bowl and the blank bowl. Half of the trials were target (T) trials, and the remaining half were distractor (D) trials presented in a pseudorandom order. Rats had to use the crossmodal features of the presented odor-texture bowl to determine the correct bowl choice.

Table 1. List of odorants and textures from which the experimental stimuli were created

\begin{tabular}{ll}
\hline Aromatherapy oils & Textures \\
\hline Patchouli & Masking tape \\
Geranium & Faux fur \\
Ylang ylang & Sandpaper \\
Lavender & Foam \\
Tea-tree & Cardboard \\
Tangerine & Packing tape \\
Almond & Velcro \\
Vanilla & Suede \\
Strawberry & Terry cloth \\
Jasmine & Duct tape \\
Bergamot & Chiffon \\
Cinnamon & Velvet \\
Chamomile & Silk \\
\hline
\end{tabular}

To test this hypothesis, we bilaterally lesioned the NBM of rats using the cholinergic-selective immunotoxin, 192 IgG-saporin, and compared their ability to that of sham-lesioned rats to (1) retrieve a crossmodal FC stimulus set learned before surgery; (2) acquire a novel crossmodal FC stimulus set; and (3) acquire two crossmodal FS stimulus sets, one of greater difficulty than the other, but neither requiring feature binding. We used the same forced-choice digging paradigm as that used in our previous pharmacology work (Botly and De Rosa, 2007, 2008).

\section{Materials and Methods \\ Subjects}

Subjects were 20 experimentally naive male Long-Evans rats (Charles River) that weighed 211-230 $\mathrm{g}$ at the start of the experiment. Rats were housed individually in 45 -cm-long $\times 25$ $\mathrm{cm}$-wide plastic tub cages and maintained on a reversed $12 \mathrm{~h} \mathrm{light/dark} \mathrm{cycle} \mathrm{(lights} \mathrm{off} \mathrm{at} \mathrm{8:00}$ A.M.) with testing occurring during the dark phase (between 10:00 A.M. and 4:00 P.M.). Rats were maintained at $90 \%$ of ad libitum freefeeding weight for the duration of the experiment. This study was approved by the University of Toronto's Institutional Animal Care Committee.

\section{Apparatus}

The training environment was a black Plexiglas chamber $30.5 \mathrm{~cm}$ high $\times 76.2 \mathrm{~cm}$ long $\times 45.7$ $\mathrm{cm}$ wide. A black slide-in-door of the same material separated the chamber into two compartments (Fig. 1). The door was positioned 25.4 $\mathrm{cm}$ from the back wall of the chamber creating a "start box" ( $25.4 \mathrm{~cm}$ long $\times 45.7 \mathrm{~cm}$ wide $)$ and a "testing arena" $(50.8 \mathrm{~cm}$ long $\times 45.7 \mathrm{~cm}$ wide). The experimental room was illuminated by a single 60 -watt light bulb. The digging apparatus was positioned on a table next to a computer equipped with speakers that emitted white noise and ambient voices to mask any extraneous noises.

\section{Odor-texture digging bowls}

All digging bowls $(8 \mathrm{~cm}$ deep $\times 4 \mathrm{~cm}$ diameter $)$ were painted matte black and attached to heavy $10 \mathrm{~cm}^{2} 4$-mm-thick black metal bases. The blank bowl had no additional sensory attributes. In contrast, the outside surface, rim, and metal base of each of the odor-texture bowls was covered by a textured cloth using silicon glue, which allowed for the easy removal of the textures. To minimize the tendency for rats to use visual cues to discriminate the textures, all textures were various shades of brown. Glued to the bottom interior surface of each odortexture bowl was a small metal cap ( $8 \mathrm{~cm}$ in diameter $\times 1.1 \mathrm{~cm}$ high) containing cotton gauze. Each cap contained 32 small holes $3 \mathrm{~mm}$ in diameter. At the beginning of each training day and after the first four rats completed their sessions, the gauze was reinjected with $0.1 \mathrm{ml}$ of the appropriate scented undiluted aromatherapy oil (Aveda; The Body Shop). However, before beginning each rat's sessions, the strength of the odor in each of the digging bowls was checked by the experimenter and additional aromatherapy oil injected if necessary to ensure consistent odor potency across rats. Before bowls were reused for novel stimuli, they were thoroughly soaked in rubbing alcohol for $3 \mathrm{~d}$ and spray painted to remove any lingering odor. Table 1 lists the odorants and textures from which the experimental stimuli were created.

\section{Forced-choice digging paradigm}

Figure 1 illustrates the two different trial types: (1) target and distractor and (2) a typical session. On every trial, rats were simultaneously presented with two digging bowls in the testing arena: an odor-texture bowl and a blank bowl, both of which were filled approximately three-quarters of the way full of the granular commercial bedding Bed-o'cobs (The Andersons). On target trials, the reward (half piece of Kellogg's Froot 


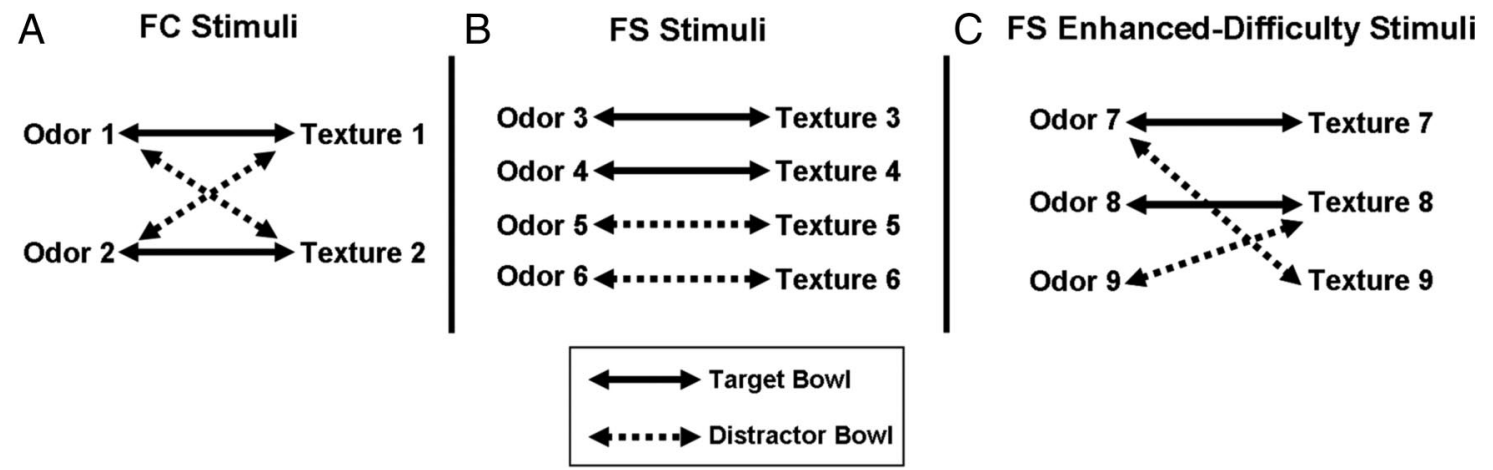

Figure 2. $\quad \boldsymbol{A}-\boldsymbol{C}$, Illustration of the features defining the $\mathrm{FC}(\boldsymbol{A})$, FS (B), and FS enhanced-difficulty $(\boldsymbol{C})$ stimuli. Solid lines indicate pairings of crossmodal features in target bowls, and dashed lines indicate pairings of crossmodal features in distractor bowls. For the FC stimuli, feature binding was required for correct bowl selection, as each individual odor and texture were associated with both a target and a distractor bowl. For both types of FS stimuli, feature binding was not required, as rats could rely on a single feature (odor or texture) for correct bowl selection. However, for the FS enhanced-difficulty stimuli, rats had the additional requirement of learning when to rely on odor and when to rely on texture to determine the correct bowl choice as one odor (odor 7) and one texture (texture 8) were associated with both a target and a distractor bowl.

Loops cereal) was buried in the odor-texture bowl, and on distractor trials, the reward was buried in the blank bowl. Rats were trained to dig in only one bowl per trial, thus requiring that they extract a rule for bowl selection. Finely ground pieces of Froot Loops cereal were added to the bedding of all bowls to mask the location of the reward. The Froot Loop reward was always buried approximately two-thirds of the way down into the bedding.

\section{Baseline stimuli}

Because of the complexity of our forced-choice digging paradigm, rats were first introduced to the different responses needed for the two trial types, target and distractor, without the need for feature binding using two baseline odor-texture bowls. Rats could use a single odor or texture or the distinct combination of the two features to determine the correct bowl choice as each baseline stimulus was characterized by a distinct odor and texture. One of the odor-texture baseline bowls was designated a target bowl (B1), and the other odor-texture baseline bowl was designated a distractor bowl (B2). Although an odor-texture bowl and the blank bowl were present on every trial, on target trials, the rat had to dig in the odor-texture bowl, and on distractor trials, the rat had to dig in the blank bowl to retrieve the reward. The same two baseline odor-texture bowls were used throughout the experiment to measure the effect of lesion on general stimulus discrimination and digging performance.

\section{FC stimuli}

Each FC stimulus set contained four conjunction odor-texture bowls and the blank bowl. Two of the four odor-texture bowls were designated target bowls (T1 and T2) and the remaining two odor-texture bowls were designated distractor bowls (D1 and D2). As illustrated by Figure 2A, crossmodal binding of odors and textures was required to determine the correct bowl choice, as each individual odor and texture was associated with both a target and a distractor bowl such that no single odor or texture could be used for correct bowl selection. That is, the crossmodal features of the target and distractor bowls overlapped.

\section{FS stimuli}

The FS stimulus set contained four nonconjunction odor-texture bowls and the blank bowl. Two of the four odor-texture bowls were designated target bowls (T1 and T2) and the remaining two odor-texture bowls were designated distractor bowls (D1 and D2). As illustrated by Figure $2 B$, feature binding was not required to determine the correct bowl choice, as each individual odor and texture was associated with either a target or a distractor bowl such that rats could use a single odor or texture or the distinct combination of the two features for correct bowl selection. That is, the crossmodal features of the target and distractor bowls were distinct and did not overlap.

\section{FS enhanced-difficulty stimuli}

The FS enhanced-difficulty stimulus set contained four nonconjunction odor-texture bowls and the blank bowl. Two of the four odor-texture bowls were designated target bowls (T1 and T2), and the remaining two odor-texture bowls were designated distractor bowls (D1 and D2). As was the case for the FS stimuli, feature binding was not required, as rats could use a single odor or texture or the distinct combination of the two features to determine the correct bowl choice. However, as illustrated by Figure $2 C$, the difficulty of the FS enhanced-difficulty stimuli was greater than that of the FS stimuli because rats had the additional requirement of learning when to rely on odor and when to rely on texture to determine the correct bowl choice. That is, one odor (odor 7) and one texture (texture 8 ) were associated with both a target and a distractor bowl, forcing the rats to use the particular crossmodal features of the odortexture bowl to determine whether to use odor or texture for correct bowl selection.

\section{Presurgical training procedures}

Habituation. The testing arena was baited with two whole Froot Loops, and rats were allowed to explore the apparatus until they consumed at least one of the treats.

Pretraining. For $2 \mathrm{~d}$, rats were trained to dig for a single whole Froot Loop buried at the bottom of an aluminum metal food bowl in their home cages. Sessions lasted $\sim 1 \mathrm{~h}$. The bowls used in their home cages were different from those used during subsequent experimental training in the testing arena: they clipped onto the side of their home cage and measured $4 \mathrm{~cm}$ deep and $8 \mathrm{~cm}$ in diameter.

General forced-choice digging procedures. At the start of every session, rats were placed in the start box of the apparatus with the sliding door closed. During this time, the appropriate bowl was baited with a single half Froot Loop and placed beside the unrewarded bowl in the testing arena of the apparatus. The blank bowl was always positioned in the far left corner of the chamber with the odor-texture bowl directly in front and flush against the blank bowl to counter the rats' natural strategy to first dig in the blank bowl and then over trials to acquire when to dig in the odor-texture bowl. The sliding door was then lifted, and the rat was allowed to make a bowl choice, after which it was gently guided back to the start box and allowed to eat the Froot Loop (if obtained) with the door closed. A choice was defined as a dig if one or both paws displaced the bedding of the chosen bowl or if a rat put its nose half-way down into the bedding. Rats remained in the start box between trials while the bowls were rebaited and replaced, which took on average $30 \mathrm{~s}$. Rats received one session per day. The first few sessions were always discovery sessions, during which rats were allowed to make as many choices as necessary to find the buried reward, but only the first bowl choice counted toward accuracy. During all remaining sessions, rats were only allowed to make a single bowl choice. If rats did not make a choice within 2-3 min, then the Froot Loop was removed and placed on top of the bedding of the rewarded bowl for the rat to find and consume in the start box. In between sessions, any bedding that accumulated in the apparatus was vacuumed up and the walls and floor of the apparatus were wiped down with rubbing alcohol. 
Acquisition of baseline stimuli. Each session consisted of 14 trials, half of which were target trials and half of which were distractor trials. Within a session, trials were presented in a pseudorandom order, such that no more than three consecutive trials were of the same type (target or distractor). The first three sessions were discovery sessions. Baseline training continued until all rats reached a criterion of at least six of seven correct target and six of seven correct distractor trials for at least two nonconsecutive sessions.

Acquisition of learning-to-learn FC stimuli. After the final baseline training session, in the subsequent session, rats began feature binding training using the learning-to-learn FC stimulus set. FC sessions consisted of 16 trials, the first four of which were baseline trials (two target and two distractor trials) identical to those described above. The remaining 12 trials of a session were six target trials (T1, three; $\mathrm{T} 2$, three) and six distractor trials (D1, three; D2, three) presented in a pseudorandom order, such that no more than three consecutive trials of the same type (target or distractor) occurred in a session. The first three sessions were discovery sessions, and only during training on this initial learning-tolearn stimulus set did rats receive correction trials in which an incorrect response was always followed by the same trial until a correct choice was made. Correction trials did not count toward accuracy.

To ensure that stable and high levels of performance were attained on the FC task before surgery, a performance criterion of five of six correct FC target and five of six correct FC distractor trials was used. Training continued until all rats reached criterion performance for at least 10 consecutive sessions.

\section{Surgery}

Rats were assigned to one of two surgical groups, sham-lesion $(n=8)$ or NBM-lesion $(n=12)$, equating the groups for presurgical baseline and FC performance. One rat became ill and was killed before surgery, reducing the NBM-lesion sample size to 11. Surgeries were performed under aseptic conditions. Rats were anesthetized with isoflurane (approximate maintenance dose was $2 \%$ with $1 \mathrm{~L} / \mathrm{min}$ of oxygen). A subcutaneous injection of the analgesic buprenorphine $(0.03 \mathrm{mg} / \mathrm{kg})$ and an intraperitoneal injection of atropine $(0.05 \mathrm{mg} / \mathrm{kg})$ were delivered immediately before surgery, the latter of which served to prevent fluid buildup in the lungs. Four $1.0 \mathrm{~mm}$ holes were drilled at the following stereotaxic coordinates relative to bregma and the surface of the skull (Paxinos and Watson, 1998): anterior NBM: anterior-posterior (AP) $-0.8 \mathrm{~mm}$, medial-lateral (ML) $\pm 2.5 \mathrm{~mm}$, dorsal-ventral (DV) $-8.2 \mathrm{~mm}$; posterior NBM: AP $-1.6 \mathrm{~mm}, \mathrm{ML} \pm 2.5 \mathrm{~mm}, \mathrm{DV}-7.6 \mathrm{~mm}$. It should be noted that our anterior lesion site bordered the most dorsal and lateral extent of the substantia innominata; however, we refer to our lesions as NBM lesions, given that our injection sites were primarily restricted to the anatomical confines of the NBM as verified by immunohistochemical analyses of the extent of our lesions (see Results). There were a total of four intraparenchymal injection sites, two per hemisphere, of $0.2 \mu \mathrm{l}$ sterile $0.1 \mathrm{M}, \mathrm{pH} 7.4$, PBS (sham-lesion) or $0.2 \mu \mathrm{g} / \mu \mathrm{l} 192 \mathrm{IgG}$-saporin (Advanced Targeting Systems; lot \#41-105) dissolved in sterile $0.1 \mathrm{M}, \mathrm{pH} 7.4$, PBS through a 26gauge Hamilton syringe at $0.1 \mu \mathrm{l} / \mathrm{min}$. The needle was left in place for $3 \mathrm{~min}$ after each injection. Choice of coordinates for lesioning and dose of immunotoxin were based on pilot surgeries performed in our laboratory. The body temperature of each rat was maintained with a homeothermic blanket throughout the surgery. After the injections were complete, a small piece of sterile gelfoam was applied over the exposed skull to control any bleeding, and the wound was closed with staples and EMLA topical analgesic ointment (2.5\% lidocaine and $2.5 \%$ prilocaine) was liberally applied around the staples. To prevent dehydration, rats were given normal saline $(0.9 \% \mathrm{NaCl} ; 2$ $\mathrm{ml} / 100 \mathrm{~g}$ body weight; s.c.) immediately after surgery. All rats received a minimum of $14 \mathrm{~d}$ of recovery with ad libitum food and water before being food restricted for subsequent testing.

\section{Postsurgical testing procedures}

Experimenters were blind to the surgical group of the animals. All postsurgical FC and FS sessions consisted of 16 trials, the first four of which were baseline trials (two target and two distractor trials) identical to those described above to measure the effect of lesion on general stimulus discrimination and digging performance. The remaining 12 trials of a ses- sion were six target trials (T1, three; $\mathrm{T} 2$, three) and six distractor trials (D1, three; D2, three) presented in a pseudorandom order, such that no more than three consecutive trials of the same type (target or distractor) occurred in a session. The same odor-texture pairings were used for rats in both the NBM- and sham-lesioned groups across all stimulus sets to ensure that differences between particular odors and textures did not influence any effect of lesion.

Retrieval of baseline stimuli. To ensure that rats could still perform the forced-choice digging task and discriminate the odors and textures after surgery, rats retrieved the baseline stimuli they had acquired before surgery. Baseline retrieval comprised two high-accuracy sessions.

Retrieval of FC stimuli. After the final baseline retrieval session, in the subsequent session, rats began retrieval of the learning-to-learn FC stimulus set they had acquired before surgery. FC retrieval sessions continued until all rats reached asymptotic performance, which took six sessions.

Acquisition of FC stimuli. After the final FC retrieval session, in the subsequent session, rats began acquisition of novel FC stimuli. FC acquisition sessions continued until all rats reached asymptotic performance, which took 16 sessions.

Acquisition of FS stimuli. After the final FC acquisition session, in the subsequent session, rats began acquisition of the FS stimuli. FS acquisition sessions continued until all rats reached asymptotic performance, which took 12 sessions.

Acquisition of FS enhanced-difficulty stimuli. After the final FS acquisition session, in the subsequent session, rats began acquisition of the FS enhanced-difficulty stimuli. FS enhanced-difficulty acquisition sessions continued until all rats reached asymptotic performance, which took 12 sessions.

\section{Histological analyses}

Rats were deeply anesthetized with sodium pentobarbital ( $60 \mathrm{mg} / \mathrm{kg}$, i.p.) and transcardially perfused with $\sim 150 \mathrm{ml}$ of ice-cold normal saline followed by $\sim 150 \mathrm{ml}$ of ice-cold $4 \%$ paraformaldehyde. Brains were extracted and immediately postfixed in $4 \%$ paraformaldehyde for $2 \mathrm{~h}$ at $4^{\circ} \mathrm{C}$ and then transferred to a solution of $20 \%$ sucrose in PBS (0.1 M; pH 7.4) and stored for 2 weeks at $4^{\circ} \mathrm{C}$. Brains were sectioned at a thickness of 60 $\mu \mathrm{m}$ using a cryostat equipped with a freezing-sliding microtome (Leica Microsystems). Adjacent sections were used for staining for acetylcholinesterase (AChE) histochemistry, choline acetyltransferase (ChAT) immunohistochemistry, and parvalbumin immunohistochemistry. AChE histochemistry was performed according to the method described by Paxinos and Watson (2007) and was used to confirm cholinergic fiber loss in target neocortical structures of the NBM. ChAT and parvalbumin immunohistochemistry were performed according to methods described in the work of Baxter et al. (1995) and De Rosa et al. (2001) to assess the extent of cholinergic and GABAergic cell body loss in the NBM and medial septum/vertical limb of the diagonal band of Broca (MS/VDB), respectively. After completion of all histological assays, brain slices were mounted on slides, dehydrated, and cleared using an ascending ethanol and xylene series, coverslipped with the histological mountant distryrene plasticizer xylene (DPX), and examined under a Leica light microscope (DM4000B).

\section{Histological quantification}

Cell counting. ChAT- and parvalbumin-immunoreactive cells were counted bilaterally in the NBM and MS/VDB as delineated by Paxinos and Watson (2007) for each rat across brain sections $300 \mu \mathrm{m}$ apart using a Leica light microscope (DM4000B) and Openlab image analysis software (Quorum Technologies). For the NBM, cell counts were taken from brain sections at approximately the following AP coordinates relative to bregma: $\mathrm{AP}=-0.84 \mathrm{~mm},-1.32 \mathrm{~mm}$, and $-1.56 \mathrm{~mm}$. The rectangular outlines superimposed on the rat brain coronal schematics depicted in Figure 3 (Paxinos and Watson, 2007) delineate the NBM cell-counting frames used for histological quantification. For the MS/VDB, approximately the following AP coordinates relative to bregma were used for cell counting: $\mathrm{AP}=+0.84 \mathrm{~mm},+0.72 \mathrm{~mm}$, and $+0.60 \mathrm{~mm}$. The rectangular outlines superimposed on the rat brain schematics depicted in Figure 4 (Paxinos and Watson, 2007) delineate the MS/VDB cell-counting frames used for histological quantification. ChAT- and parvalbumin- 


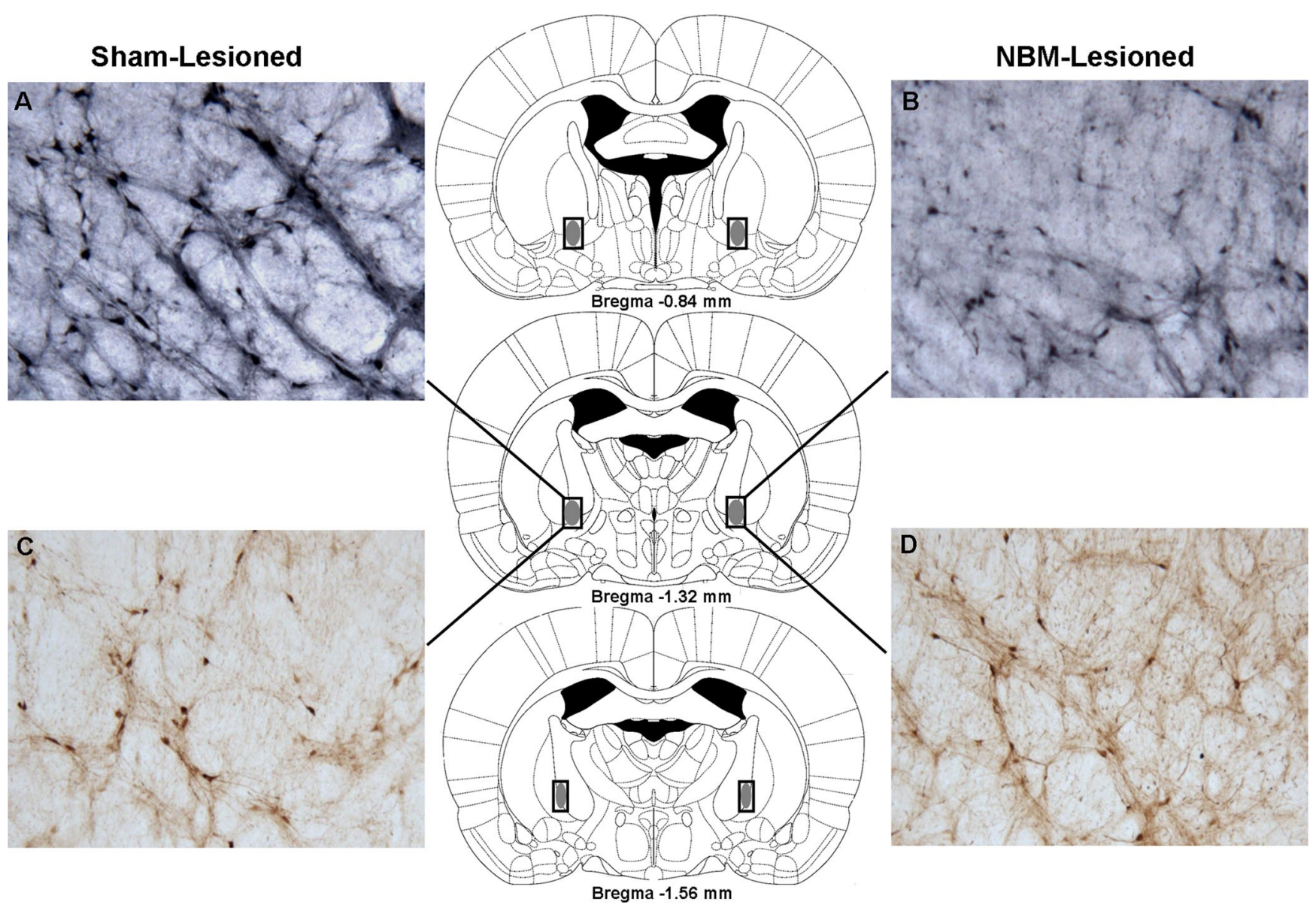

Figure 3. Choline acetyltransferase and parvalbumin immunohistochemistry of the NBM. $\boldsymbol{A}-\boldsymbol{D}$, Panels (10× magnification) depict ChAT-immunoreactive cells $(\boldsymbol{A}, \boldsymbol{B})$ and parvalbuminimmunoreactive cells $(\boldsymbol{C}, \boldsymbol{D})$ in the NBM of a typical sham-lesioned $(\boldsymbol{A}, \boldsymbol{C})$ and NBM-lesioned $(\boldsymbol{B}, \boldsymbol{D})$ rat. A loss of ChAT-immunoreactive cells $(\boldsymbol{B})$, but not parvalbumin-immunoreactive cells $(\boldsymbol{D})$, is apparent in the NBM-lesioned rat. The rectangular outlines superimposed on the rat brain coronal schematics delineate the NBM cell-counting frames, and the solid gray fill illustrates the typical extent of ChAT-immunoreactive cell loss. Rat brain schematics were adapted from Paxinos and Watson (2007) and displayed coordinates refer to the anterior-posterior plane.

immunoreactive cells were easily identifiable and characterized by relatively large cell bodies with several extending dendrites, and we only counted cells that were well distinguishable (i.e., well defined borders) from the background.

AChE densitometry. To quantify the reduction of cortical cholinergic input induced by our NBM cholinergic lesions, estimates of optical density in the frontal and parietal cortices and hippocampus were obtained from photomicrographs of AChE-stained brain sections using the software package Scion (Scion). For each rat, optical density values obtained from the three target brain regions were then normalized to raw striatal optical density values to eliminate the potential influence of different staining intensities across animals [see similar method used by Vuckovich et al. (2004)]. Raw optical density values from the striatum did not differ between the NBM- and sham-lesioned groups (see Results). The rectangular outlines superimposed on the rat brain schematics depicted in Figure 5 (Paxinos and Watson, 2007) delineate the boundaries used for obtaining optical density values in the frontal and parietal cortices and hippocampus.

\section{Statistical analysis}

All statistical analyses were conducted using SPSS v.14 with an $\alpha$-level of 0.05 .

\section{Results}

\section{Presurgical training}

Thirteen training sessions were required for all rats to reach criterion performance on the baseline task, and 79 training sessions were required for all rats to reach criterion performance on the FC task using the learning-to-learn FC stimulus set.

\section{Histological analyses}

Of the 20 rats, one became ill and was killed before surgery, and two sham-lesioned rats became ill and were killed after surgery. Postmortem assessment revealed that all three of these rats had become diabetic. Accordingly, behavioral and histological data from these three rats were removed from statistical analyses for a total of 17 rats. Quantitative histological analyses revealed that two NBM-lesioned rats had statistically insufficient reductions of ChAT-immunoreactive cells in the NBM as indicated by a ChATimmunoreactive cell count greater than two SDs above the mean of the NBM-lesioned group and nonsignificantly different from the mean of the sham-lesioned group. Moreover, the AChE optical density values obtained from the frontal and parietal cortices of these two animals were nonsignificantly different from the means of the sham-lesioned group. Accordingly, behavioral and histological data from these two rats were removed from statistical analyses. The sham-lesion sample was thus reduced to six, and the NBM-lesion sample size was reduced to nine.

\section{Cell counting}

There was significant depletion of ChAT-immunoreactive cells in the NBM of the NBM-lesioned rats relative to sham-lesioned rats 


\section{Sham-Lesioned}
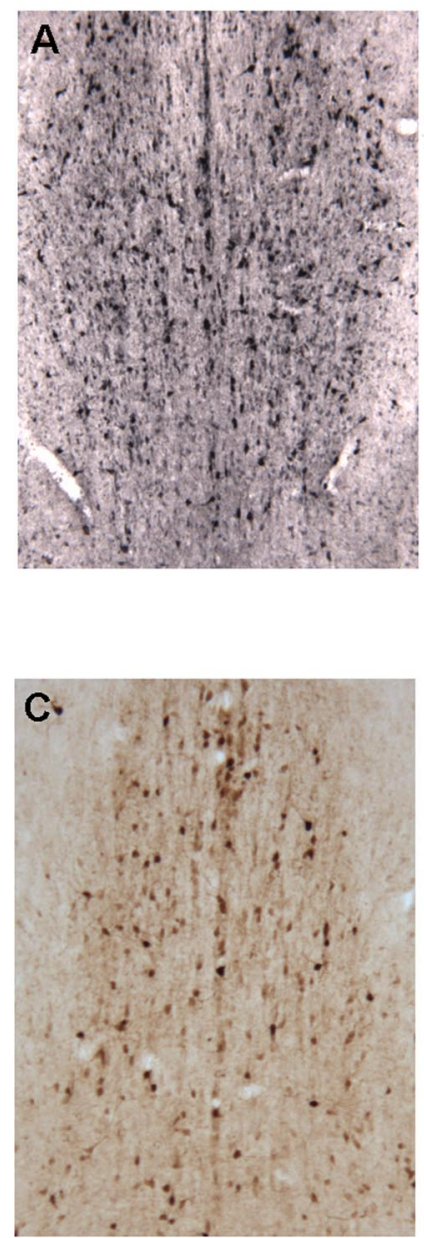

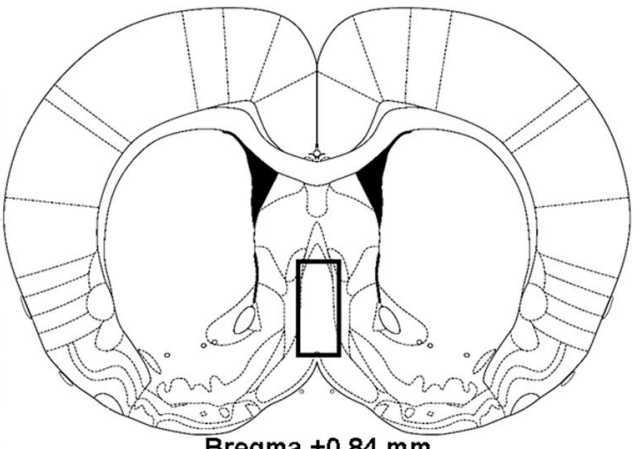

Bregma $+0.84 \mathrm{~mm}$

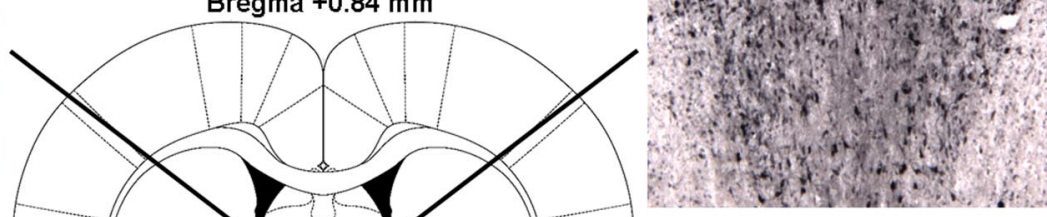

NBM-Lesioned

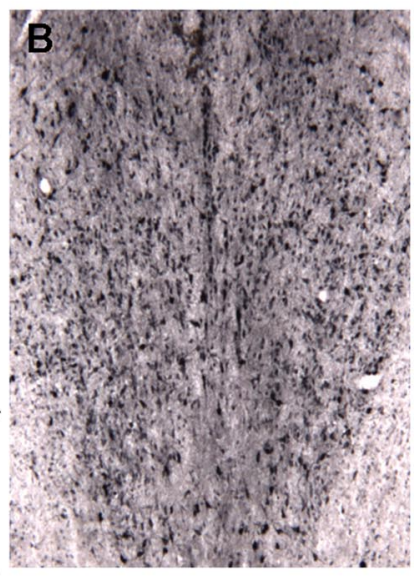

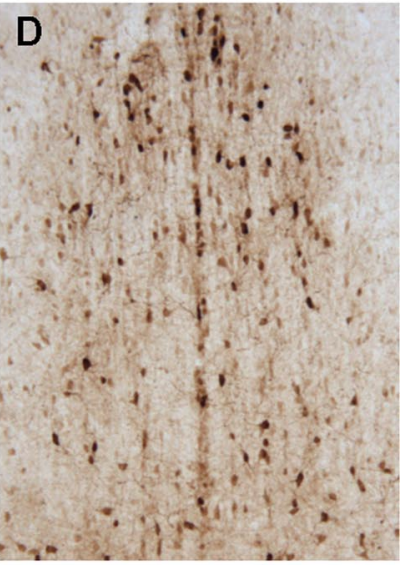

Figure 4. Choline acetyltransferase and parvalbumin immunohistochemistry of the MS/VDB. $\boldsymbol{A}-\boldsymbol{D}$, Panels (10× magnification) depict ChAT-immunoreactive cells ( $\boldsymbol{A}, \boldsymbol{B})$ and parvalbuminimmunoreactive cells $(\boldsymbol{C}, \boldsymbol{D})$ in the MS/VDB of a typical sham-lesioned $(\boldsymbol{A}, \boldsymbol{C})$ and NBM-lesioned $(\boldsymbol{B}, \boldsymbol{D})$ rat. No loss of ChAT-immunoreactive cells $(\boldsymbol{B})$ or parvalbumin-immunoreactive cells $(\boldsymbol{D})$ is apparent in the NBM-lesioned rat. The rectangular outlines superimposed on the rat brain coronal schematics delineate the MS/VDB cell-counting frames. Rat brain schematics were adapted from Paxinos and Watson (2007) and displayed coordinates refer to the anterior/posterior plane.

(Fig. 3), whereas an equivalent number of ChAT-immunoreactive cells were observed in the neighboring MS/VDB nuclei of both groups of rats (Fig. 4). Independent $t$ tests confirmed that there were significantly fewer ChAT-immunoreactive cells in the NBM of NBM-lesioned compared with sham-lesioned rats $\left[t_{(13)}=15.05\right.$, $p<0.001$; mean $(\mathrm{M})_{\text {Sham }}=405.50, \mathrm{SD}=41.27 ; \mathrm{M}_{\mathrm{NBM}}=129.56$, $\mathrm{SD}=30.05]$. However, there was no significant difference between the two groups of rats in the number ChAT-immunoreactive cells in the MS/VDB $\left(t<2.0 ; \mathrm{M}_{\text {Sham }}=251.67, \mathrm{SD}=19.12 ; \mathrm{M}_{\mathrm{NBM}}=\right.$ $238.11, \mathrm{SD}=11.78)$. An equivalent number of parvalbuminimmunoreactive cells were observed in the NBM (Fig. 3) and MS/ VDB (Fig. 4) of both sham- and NBM-lesioned rats. Independent $t$ tests confirmed that there were no significant differences, $t<1.0$, between the two groups of rats in the number of parvalbuminpositive cells in the NBM $\left(\mathrm{M}_{\text {Sham }}=161.33, \mathrm{SD}=28.24 ; \mathrm{M}_{\mathrm{NBM}}=\right.$ $161.44, \mathrm{SD}=24.13)$ and $\mathrm{MS} / \mathrm{VDB}\left(\mathrm{M}_{\mathrm{Sham}}=228.33, \mathrm{SD}=27.28\right.$; $\left.\mathrm{M}_{\mathrm{NBM}}=221.22, \mathrm{SD}=28.90\right)$.

\section{AChE densitometry}

AChE staining revealed a loss of cholinergic fibers in the frontal and parietal cortices but not the hippocampus of NBM-lesioned rats relative to sham-lesioned rats (Fig. 5). This was confirmed by independent $t$ tests comparing the optical density values (normalized to raw striatal optical density values) from the three target brain regions between the two groups of rats. The raw optical density values from the striatum (used for normalization) did not differ between the two groups $\left(t<1.0 ; \mathrm{M}_{\text {Sham }}=240.13, \mathrm{SD}=4.91 ; \mathrm{M}_{\mathrm{NBM}}=\right.$ $240.05, \mathrm{SD}=6.77)$. There was significantly less AChE reactivity as measured by optical density in the frontal $\left(t_{(13)}=9.09, p<0.001\right.$; $\left.\mathrm{M}_{\text {Sham }}=0.53, \mathrm{SD}=0.03 ; \mathrm{M}_{\mathrm{NBM}}=0.38, \mathrm{SD}=0.03\right)$ and parietal $\left(t_{(13)}=6.59, p<0.001 ; \mathrm{M}_{\text {Sham }}=0.47, \mathrm{SD}=0.02 ; \mathrm{M}_{\mathrm{NBM}}=0.38\right.$, $\mathrm{SD}=0.03)$ cortices of NBM-lesioned compared with sham-lesioned rats. However, there was no significant difference between the two groups of rats in AChE reactivity in the hippocampus as measured by optical density $\left(t<1.0 ; \mathrm{M}_{\text {Sham }}=0.60, \mathrm{SD}=0.05 ; \mathrm{M}_{\mathrm{NBM}}=0.61\right.$, $\mathrm{SD}=0.06)$

\section{Postsurgical testing}

Retrieval of baseline stimuli

Rats in both lesion conditions maintained very high accuracies on the baseline stimuli during the first postsurgical retrieval session $\left(\mathrm{M}_{\text {Sham }}=85 \%, \mathrm{SD}=0.20 ; \mathrm{M}_{\mathrm{NBM}}=88 \%, \mathrm{SD}=0.11\right)$. An ANOVA was performed using lesion condition (sham-lesion or 


\section{Sham-Lesioned}
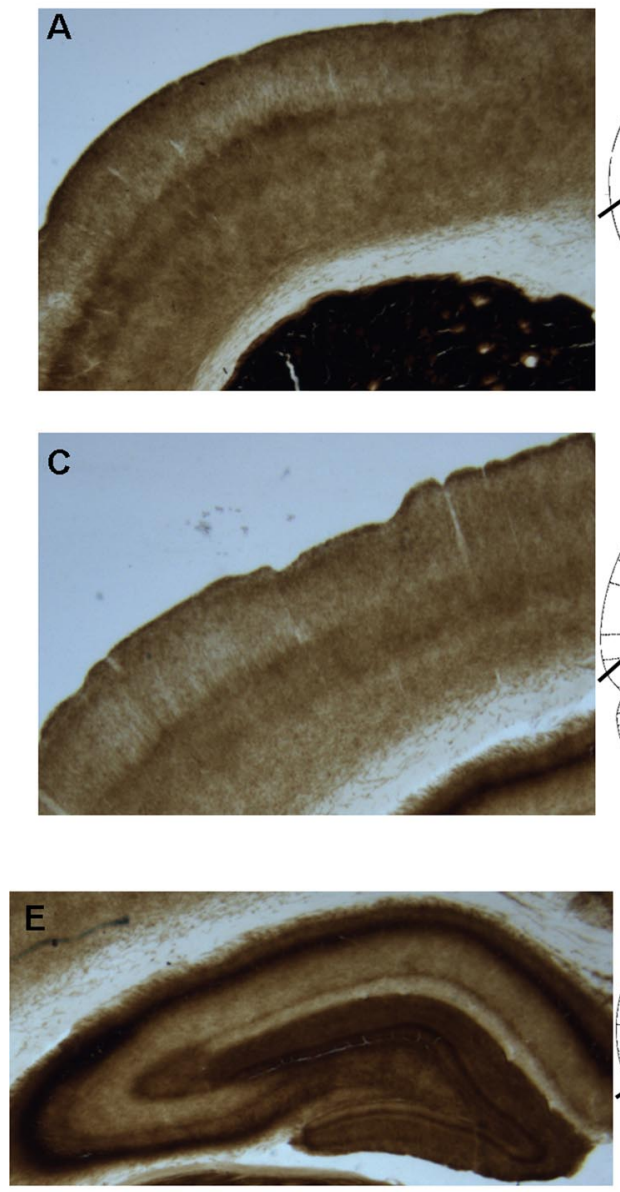

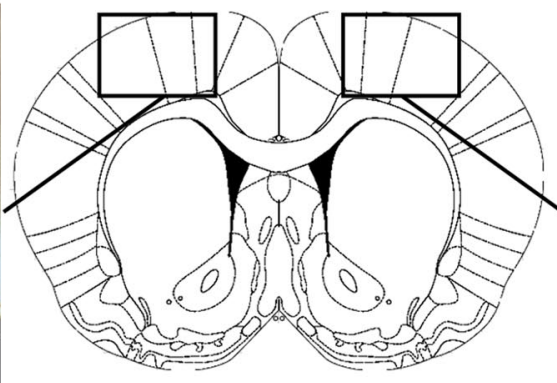

Bregma $+1.32 \mathrm{~mm}$

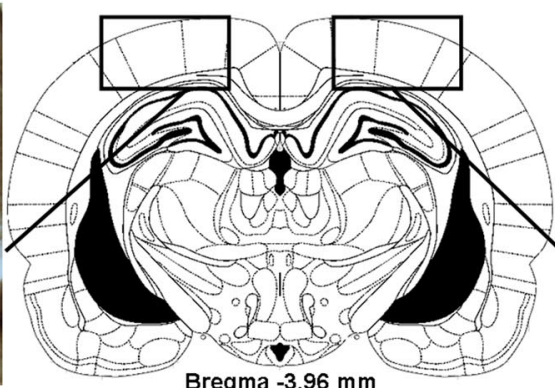

Bregma $-3.96 \mathrm{~mm}$

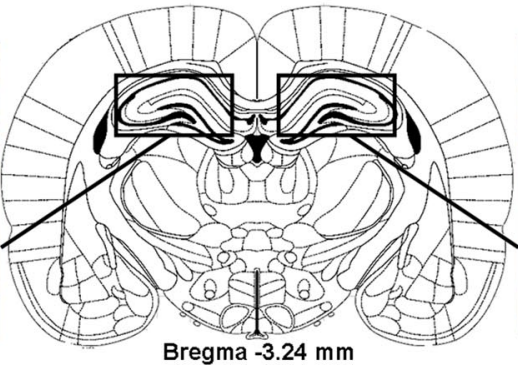

NBM-Lesioned
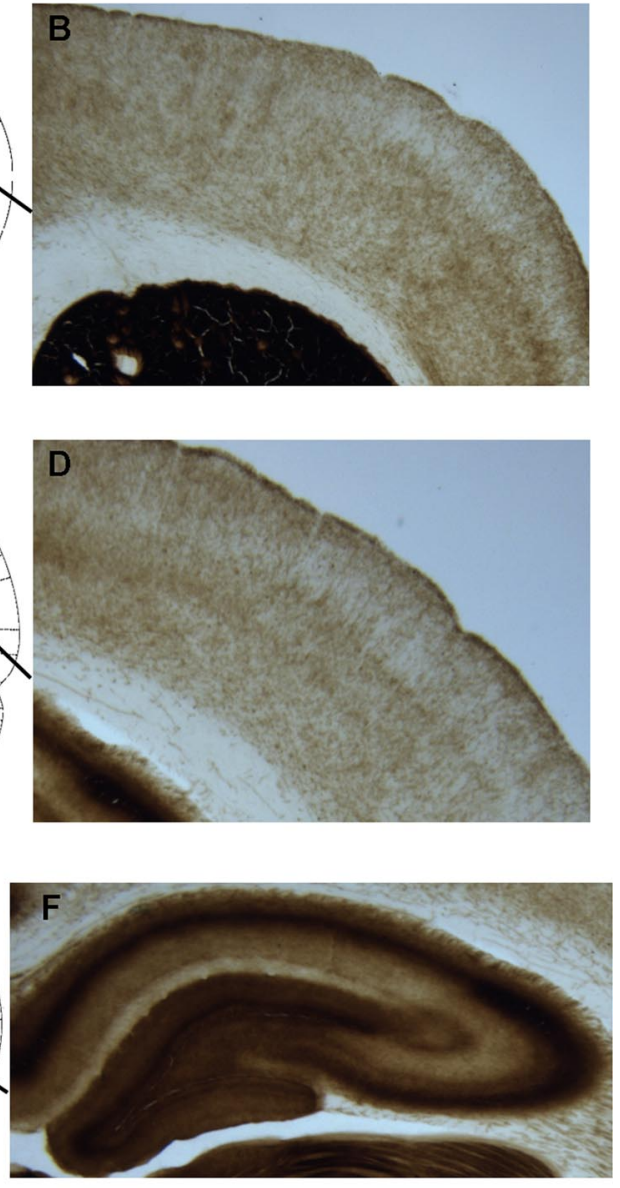

Figure 5. Acetylcholinesterase histochemistry. $\boldsymbol{A}-\boldsymbol{F}$, Panels (1.25 $\times$ magnification) depict AChE staining in the frontal cortex $(\boldsymbol{A}, \boldsymbol{B})$, parietal cortex $(\boldsymbol{C}, \boldsymbol{D})$, and hippocampus $(\boldsymbol{E}, \boldsymbol{F})$ of a typical sham-lesioned $(\boldsymbol{A}, \boldsymbol{C}, \boldsymbol{E})$ and NBM-lesioned $(\boldsymbol{B}, \boldsymbol{D}, \boldsymbol{F})$ rat. Significant depletion of AChE-positive fibers in the frontal $(\boldsymbol{B})$ and parietal $(\boldsymbol{D})$ cortices, but not the hippocampus $(\boldsymbol{F})$, is apparent in the NBM-lesioned rat. The rectangular outlines superimposed on the rat brain coronal schematics delineate the boundaries used for obtaining optical density values for AChE densitometry. Rat brain schematics were adapted from Paxinos and Watson (2007) and displayed coordinates refer to the anterior/posterior plane.

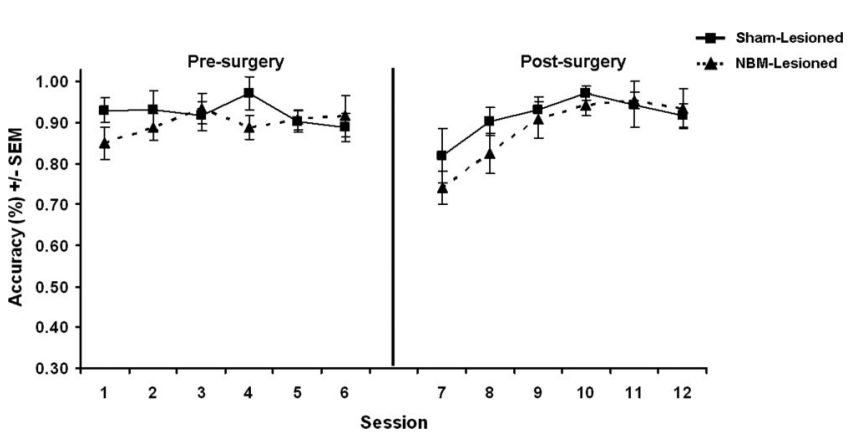

Figure 6. $\quad F C$ performance during the last six sessions before surgery and the subsequent retrieval of these same $\mathrm{FC}$ stimuli during the six postsurgery sessions.

NBM-lesion) as a between-subjects factor and session as a within-subjects factor. The ANOVA revealed no significant effect of lesion condition $\left(F<1.0, \eta^{2}=0.06\right)$, a significant effect of session $\left(F_{(1,13)}=6.38, p<0.05, \eta^{2}=0.33\right)$, and no significant interaction $\left(F<1.0, \eta^{2}=0.001\right)$.

Retrieval of FC stimuli

Figure 6 depicts the performance of rats in the NBM- and shamlesioned groups on the learning-to-learn FC stimulus set during the last six sessions before surgery and the subsequent retrieval of these same FC stimuli during the six postsurgical sessions. A two-way mixed ANOVA was performed using lesion condition (sham-lesion or NBM-lesion) as a between-subjects factor and performance period (before surgery or after surgery) and session as within-subjects factors. The ANOVA revealed no significant effect of lesion condition $\left(F<1.0, \eta^{2}=0.03\right)$ or performance period $\left(F<1.0, \eta^{2}=0.04\right)$, a significant effect of session $\left(F_{(5,65)}\right.$ $\left.=8.03, p<0.001, \eta^{2}=0.38\right)$, and a significant session $\mathrm{X}$ performance period interaction $\left(F_{(5,65)}=8.03, p<0.001, \eta^{2}=0.38\right)$. All other interactions were found to be nonsignificant $(F<2.5$, $\left.\eta^{2}<0.15\right)$. An ANOVA on baseline stimuli performance during postsurgical FC retrieval revealed nonsignificant effects of lesion condition $\left(F<1, \eta^{2}=0.04\right)$ and session $\left(F=1.0, \eta^{2}=0.08\right)$, and no significant interaction $\left(F=1.0, \eta^{2}=0.08\right)$.

\section{Acquisition of FC stimuli}

Figure $7 A$ depicts postsurgical acquisition of the FC task using a novel stimulus set with data binned into four-session blocks. An ANOVA was performed using lesion condition (sham-lesion or NBM-lesion) as a between-subjects factor and block (first or last) as a within-subjects factor. The ANOVA revealed a significant effect of lesion condition $\left(F_{(1,13)}=4.69, p=0.05, \eta^{2}=0.27\right)$, a significant effect of block $\left(F_{(1,13)}=136.22 .43, p<0.001, \eta^{2}=\right.$ 


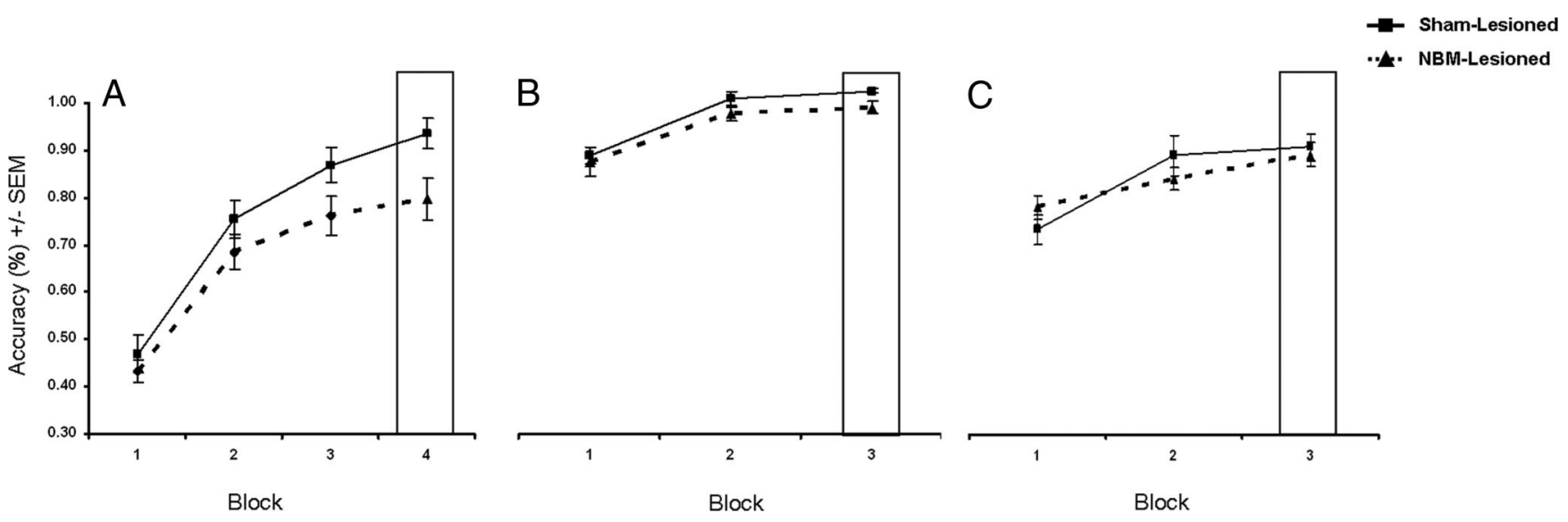

Figure 7. $\quad A-C$, Postsurgical acquisition of novel $F C(A), F S(B)$, and FS enhanced-difficulty $(\boldsymbol{C})$ stimuli. Accuracy has been binned into four-session blocks. The boxes highlight the final level of performance attained by rats on the three tasks.

Table 2. Correlations between task performance and ChAT immunoreactivity and AChE reactivity

\begin{tabular}{lllll}
\hline & $\begin{array}{l}\text { ChAT immu- } \\
\text { noreactivity }\end{array}$ & & AChE reactivity & \\
\cline { 2 - 2 } Task & NBM & & Frontal cortex & Parietal cortex \\
\hline FC retrieval & $r=0.18$ & & $r=0.20$ & $r=-0.01$ \\
FC acquisition & $r=0.56^{*}$ & & $r=0.45^{*}$ & $r=0.29^{+}$ \\
FS enhanced-difficulty acquisition & $r=0.08$ & & $r=0.19$ & $r=0.14$ \\
\hline
\end{tabular}

Correlations (Pearson's rvalues) between performance and ChAT immunoreactivity in the NBM (as measured by the number of (hAT-positive cells) and neocortical AChE reactivity (as measured by optical density). The correlations were conducted using postsurgical performance data from FC retrieval and the last block of FC and FS enhanceddifficulty acquisition. ${ }^{*} p<0.05 ;{ }^{+} p=0.10$.

$0.91)$, and no significant interaction $\left(F=2.0, \eta^{2}=0.13\right)$. An ANOVA on baseline stimuli performance during FC acquisition revealed nonsignificant effects of lesion condition $\left(F<1.0, \eta^{2}=\right.$ $0.06)$ and block $\left(F<1.5, \eta^{2}=0.09\right)$, and no significant interaction $\left(F<1.5, \eta^{2}=0.09\right)$.

Acquisition of FS stimuli

Figure $7 B$ depicts postsurgical acquisition of the FS task with data binned into four-session blocks. An ANOVA was performed using lesion condition (sham-lesion or NBM-lesion) as a betweensubjects factor and block (first or last) as a within-subjects factor. The ANOVA revealed no significant effect of lesion condition $\left(F<1.0, \eta^{2}=0.06\right)$, a significant effect of block $\left(F_{(1,13)}=58.82\right.$, $\left.p<0.001, \eta^{2}=0.82\right)$, and no significant interaction $\left(F<1.0, \eta^{2}\right.$ $=0.03)$. An ANOVA on baseline stimuli performance during FS acquisition revealed nonsignificant effects of lesion condition $\left(F<1.5, \eta^{2}=0.10\right)$ and block $\left(F<2.5, \eta^{2}=0.15\right)$, and no significant interaction $\left(F<2.0, \eta^{2}=0.01\right)$.

Acquisition of FS enhanced-difficulty stimuli

Figure $7 C$ depicts postsurgical acquisition of the FS enhanceddifficulty task with data binned into four-session blocks. An ANOVA was performed on the acquisition data using lesion condition (sham-lesion or NBM-lesion) as a between-subjects factor and block (first or last) as a within-subjects factor. The ANOVA revealed no significant effect of lesion condition $\left(F<1.0, \eta^{2}=\right.$ $0.02)$, a significant effect of block $\left(F_{(1,13)}=38.64, p<0.001, \eta^{2}=\right.$ $0.75)$, and no significant interaction $\left(F<2.0, \eta^{2}=0.13\right)$. An ANOVA on baseline stimuli performance during FS enhanceddifficulty acquisition revealed nonsignificant effects of lesion condition $\left(F<1.0, \eta^{2}=0.001\right)$ and block $\left(F<1.0, \eta^{2}=0.06\right)$, and no significant interaction $\left(F<1.0, \eta^{2}=0.02\right)$.
Indicative of its greater difficulty, sham-lesioned rats performed worse on the FS enhanced-difficulty task than the FS task during acquisition. This was confirmed by performing a withinsubjects ANOVA on sham-rat acquisition data using task (FS or FS enhanced-difficulty) and block (first or last) as withinsubjects factors. The ANOVA revealed a significant effect of block $\left(F_{(1,5)}=306.82, p<0.001, \eta^{2}=0.98\right)$ and task $\left(F_{(1,5)}=31.57\right.$, $\left.p<0.01, \eta^{2}=0.86\right)$, and no significant interaction $\left(F<1.5, \eta^{2}\right.$ $=0.21)$.

\section{Correlations between task performance and ChAT immunoreactivity and $\mathrm{AChE}$ reactivity}

The results of the correlational analyses between postsurgical task performance and ChAT immunoreactivity as measured by the number of ChAT-positive cells in the NBM, and neocortical AChE reactivity as measured by the optical density of AChE staining in the frontal and parietal cortices, are shown in Table 2. Accuracy during FC acquisition significantly and positively correlated with both ChAT immunoreactivity in the NBM and with AChE reactivity in the frontal cortex. All other correlations were found to be nonsignificant ( $p>0.05$ ); however, there was a weak trend toward significance found for the positive relationship between FC acquisition and AChE reactivity in the parietal cortex. The scatterplots depicted in Figure 8 illustrate these three relationships. There were no significant correlations found between postsurgical task performance and AChE reactivity in the hippocampus $(r<0.15, p>0.15)$, ChAT immunoreactivity in the MS/VDB $(r<0.15, p>0.30)$, and parvalbumin immunoreactivity in the NBM $(r<0.23, p>0.20)$ or MS/VDB $(r<0.15$, $p>0.20)$.

\section{Discussion}

We contend that ACh provides the neuromodulatory support for the attentional processes critical for feature binding. In support of this hypothesis, previous work using systemic pharmacology demonstrated the importance of ACh to crossmodal feature binding at encoding (Botly and De Rosa, 2007), and more recent cross-species work revealed striking parallels between the effects of modulations of attention in humans and modulations of the muscarinic cholinergic system in rats on intramodal feature binding (Botly and De Rosa, 2008). However, the neural source of the cholinergic neurotransmission critical for feature binding has yet to be determined. Human neuropsychological and functional magnetic resonance imaging (fMRI) studies have impli- 
A

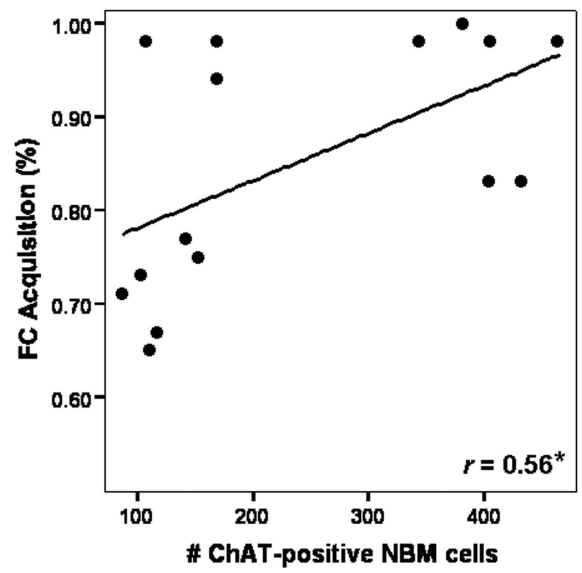

B

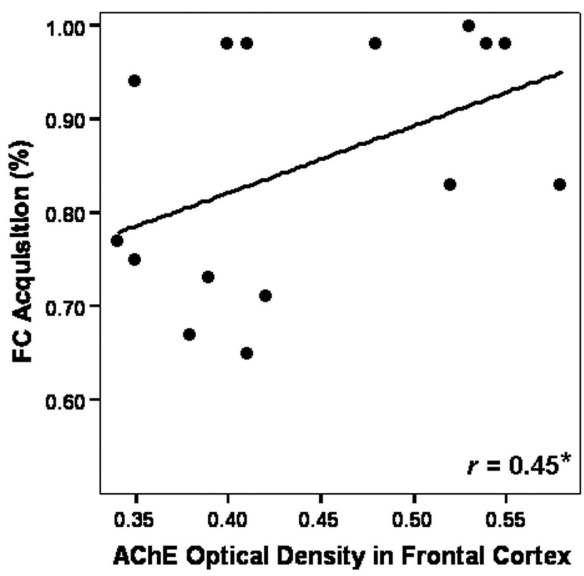

C

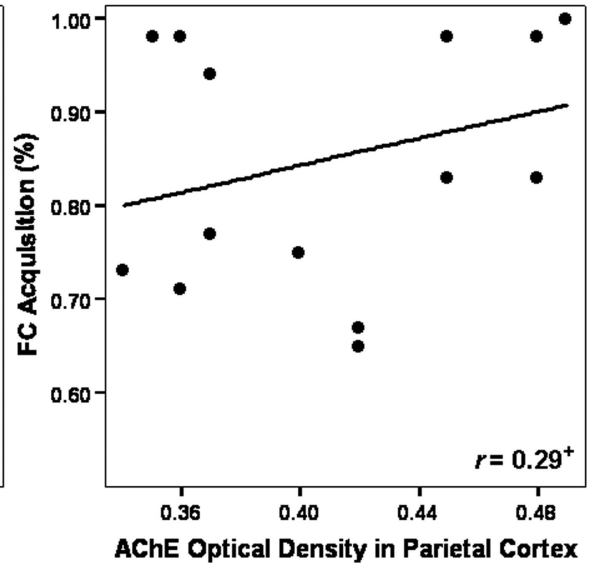

Figure 8. Scatterplots illustrating the relationships between FC acquisition and NBM ChAT immunoreactivity and neocortical AChE reactivity. $A, B$, Linear regression of the data revealed significant positive relationships between postsurgical performance during the final block of $\mathrm{FC}$ acquisition and the number of ChAT-positive cells in the NBM $(\boldsymbol{A})$ and AChE optical density in the frontal cortex $(\boldsymbol{B})$. C, A weak trend toward significance was found for the positive relationship between $\mathrm{FC}$ acquisition performance and $\mathrm{AChE}$ optical density in the parietal cortex. ${ }^{*} p<0.05$; ${ }^{+} p=0.10$.

cated frontoparietal cortices in the attentional processing necessary for feature binding (Cohen and Rafal, 1991; Corbetta et al., 1995; Friedman-Hill et al., 1995; Donner et al., 2002; Esterman et al., 2007). Cholinergic efferents originating from the NBM of the $\mathrm{BF}$, relative to other BF nuclei clustered nearby, provide $90 \%$ of the cholinergic input to the neocortex, including frontoparietal regions (Mesulam et al., 1983). We hypothesized that the NBM may be the neural source of the cholinergic neurotransmission critical for feature binding, and thus reducing cholinergic cortical afferentation via cholinergic immunotoxic lesioning of the NBM would impair feature binding in a similar manner to systemic muscarinic cholinergic blockade in rats.

The crossmodal FC and FS tasks used a forced-choice digging paradigm that required rats to decide in which of two bowls to dig to obtain a food reward. In the FC task, rats had to bind odor and texture features to determine the correct bowl choice, whereas in the FS tasks, there was no requirement to bind odor and texture features, and the rats could rely on a single feature (odor or texture). We predicted that the FS tasks would be less dependent on cortical cholinergic neurotransmission than the FC task, because processing single features should be less attentionally demanding than processing conjunctions of features. Consistent with this hypothesis, in the present study, rats with 192 IgG-saporininduced lesions of NBM were significantly impaired relative to sham-lesioned control rats at acquiring FC stimuli, while their ability to acquire FS stimuli remained intact. Additionally, the number of cholinergic (ChAT-positive) cells in the NBM and the degree of neocortical, but not hippocampal AChE reactivity, were found to positively correlate only with postsurgical FC acquisition performance, signifying the importance of neocortical cholinergic input from the NBM to feature binding at encoding.

Findings from the human cognitive literature support the low-attentional load nature of single-feature processing. Patients with attentional deficits who are impaired at encoding conjunctions of features remain fully capable of encoding single features (Cohen and Rafal, 1991; Friedman-Hill et al., 1995; Bernstein and Robertson, 1998; Foster et al., 1999; Tales et al., 2002), and human fMRI studies have revealed selective activation of parietal attentional networks during tasks requiring feature binding, but not those requiring single-feature processing (Corbetta et al., 1995; Luck and Ford, 1998; Reynolds and Desimone, 1999). More recently, it was found that transcranial magnetic stimulation of the intraparietal sulcus influenced the binding of color and form, but not the detection or processing of single features (Esterman et al., 2007).

Although the FS and FC tasks required the same number of odor-texture bowl discriminations (i.e., four), it could be argued that NBM-lesioned rats' spared acquisition of FS stimuli was simply attributable to the FS task being less difficult than the FC task. To address this potential criticism, rats acquired an FS enhanceddifficulty stimulus set in addition to acquiring an FS stimulus set. Although both FS tasks did not require feature binding, as rats could rely on a single feature (odor or texture) for correct bowl selection, the FS enhanced-difficulty stimuli required rats to also learn when to rely on odor and when to rely on texture as one odor and one texture were associated with both the correct and incorrect bowl choices. Indicative of its greater difficulty, shamlesioned rats performed worse while acquiring the FS enhanceddifficulty task than the FS task. However, there was no significant difference between the performance of rats in the two lesions groups during FS or FS enhanced-difficulty acquisition. This suggests that the feature binding requirement of the FC task increased the attentional load of the task over and above any increase induced by greater task difficulty. Findings from the human cognitive literature support this proposition: even after controlling for target-distractor similarity, which makes both FS and FC tasks more attentionally demanding, feature binding in itself requires additional attentional resources over and above those needed for making fine perceptual discriminations (Carter, 1982; Duncan and Humphreys, 1989; von Grünau et al., 1994; Foldi et al., 2005).

Last, NBM-lesioned rats were not impaired at retrieving an FC stimulus set they had acquired before surgery. This is consistent with previous pharmacological (Botly and De Rosa, 2007) and cross-species findings (Botly and De Rosa, 2008) and provides further evidence to suggest that once a conjunction stimulus is well learned, a bound and stable neural representation is formed, thereby eliminating the need for an attentionally demanding feature binding process during retrieval. Such dissociative encoding-retrieval findings are consistent with a model of cholinergic function proposing that an active cholinergic system is conducive to the encoding of new information, whereas a hypo- 
active cholinergic system is conducive to the consolidation and retrieval of previously learned information (Hasselmo and McGaughy, 2004). When there are high levels of cholinergic neurotransmission in the cortex, afferent input from external sensory stimuli is enhanced, whereas intrinsic processing within cortices is reduced.

Intrinsic processing is associated with the reactivation of neural connections representing previously learned information, and a reduction in such processing would prevent the recall of previously learned information from interfering with the encoding of new information. This model of cholinergic function is well supported by both the human and nonhuman animal literatures (Safer and Allen, 1971; Aigner et al., 1991; Anagnostaras et al., 1995; Orsetti et al., 1996; Everitt and Robbins, 1997; Miranda and Bermúdez-Rattoni, 1999; White and Ruske, 2002; Pepeu and Giovannini, 2004). For instance, scopolamine increased proactive interference from previously learned odor- and word-pair associations during the encoding of novel associations in nonhuman and human animals, respectively (De Rosa and Hasselmo, 2000; De Rosa et al., 2001; Atri et al., 2004). Furthermore, fMRI studies have shown that boosting cholinergic neurotransmission in humans with the anticholinesterase physostigmine improves memory performance by enhancing perceptual processing during the encoding stage (Furey et al., 2000).

Given past research implicating frontoparietal cholinergic input from the NBM in attention (Bucci et al., 1998; Dalley et al., 2004), we contend that it is likely cholinergic neurotransmission in a frontoparietal cortical network that is critical for feature binding, and the results of our correlational analyses between cortical AChE densitometry and postsurgical task performance are in support of this hypothesis. We found a significant positive correlation between FC acquisition and AChE reactivity in the frontal cortex and a weak trend toward significance in the parietal cortex. These findings are consistent with previous microdialysis work demonstrating positive associations between task-related attentional effort and ACh efflux in frontoparietal cortices in rats (Himmelheber et al., 2000, 2001). More recent work using a novel enzyme-based amperometric technique, which allows for the monitoring of cortical ACh release on multiple timescales in freely behaving animals, has revealed both tonic and phasic attention-dependent changes in cortical cholinergic activity (Parikh et al., 2007; Parikh and Sarter, 2008). This work demonstrated that cortex-wide tonic ACh activity was associated with efficacious attentional processing and was positively correlated with attentional effort, thus we would hypothesize greater increases in tonic frontoparietal ACh activity while rats performed our more attentionally demanding FC versus FS tasks. Additionally, we expect that these tonic ACh increases would be specific to the task-dependent regions that support the required attentional processes, and therefore would not expect to observe such increases in task-independent cortical regions such as the hippocampus. Further research is needed to determine the specific roles of tonic versus phasic attention-dependent cortical ACh activity; thus, we cannot yet speculate on what these two forms may specifically contribute to feature binding.

The present study provides insight into the functional role of the NBM and extends previous systemic pharmacology work to provide further support for a critical role for the neuromodulator $\mathrm{ACh}$ in the attention-dependent process of feature binding. Using the cholinergic immunotoxin 192 IgG-saporin to lesion the NBM of rats, it was shown that reducing cholinergic neocortical afferentation impairs crossmodal feature binding at encoding while sparing its retrieval and the acquisition of crossmodal tasks only requiring single-feature processing. Such destruction of cholinergic neurons in the NBM resulted in reduction of cholinergic input to the entire cortical mantle, and our histological densitometry analyses revealed positive relationships between postsurgical feature-binding acquisition performance and cortical AChE staining in frontoparietal regions. Accordingly, future work should concentrate on delineating the specific cortical targets cholinergic input must reach for successful feature binding to occur.

\section{References}

Aigner TG, Walker DL, Mishkin M (1991) Comparison of the effects of scopolamine administered before and after acquisition in a test of visual recognition memory in monkeys. Behav Neural Biol 55:61-67.

Anagnostaras SG, Maren S, Fanselow MS (1995) Scopolamine selectively disrupts the acquisition of contextual fear conditioning in rats. Neurobiol Learn Mem 64:191-194.

Atri A, Sherman S, Norman KA, Kirchhoff BA, Nicolas MM, Greicius MD, Cramer SC, Breiter HC, Hasselmo ME, Stern CE (2004) Blockade of central cholinergic receptors impairs new learning and increases proactive interference in a word paired-associate memory task. Behav Neurosci 118:223-236.

Baxter MG, Bucci DJ, Gorman LK, Wiley RG, Gallagher M (1995) Selective immunotoxic lesions of basal forebrain cholinergic cells: effects on learning and memory in rats. Behav Neurosci 109:714-722.

Behrmann M, Geng JJ, Shomstein S (2004) Parietal cortex and attention. Curr Opin Neurobiol 14:212-217.

Bernstein LJ, Robertson LC (1998) Illusory conjunctions of color and motion with shape following bilateral parietal lesions. Psychol Sci 9:167-175.

Botly LC, De Rosa E (2007) Cholinergic influences on feature binding. Behav Neurosci 121:264-276.

Botly LCP, De Rosa E (2008) A cross-species investigation of acetylcholine, attention, and feature binding. Psychol Sci 19:1185-1193.

Bucci DJ (2009) Posterior parietal cortex: an interface between attention and learning? Neurobiol Learn Mem 91:114-120.

Bucci DJ, Chess AC (2005) Specific changes in conditioned responding following neurotoxic damage to the posterior parietal cortex. Behav Neurosci 119:1580-1587.

Bucci DJ, Holland PC, Gallagher M (1998) Removal of cholinergic input to rat posterior parietal cortex disrupts incremental processing of conditioned stimuli. J Neurosci 18:8038-8046.

Carter RC (1982) Visual search with color. J Exp Psychol Hum Percept Perform 8:127-136.

Cohen A, Rafal RD (1991) Attention and feature integration: illusory conjunctions in a patient with parietal lobe lesion. Psychol Sci 2:106-110.

Constantinidis C (2006) Posterior parietal mechanisms of visual attention. Rev Neurosci 17:415-427.

Corbetta M, Shulman GL, Miezin FM, Petersen SE (1995) Superior parietal cortex activation during spatial attention shifts and visual feature conjunction. Science 270:802-805.

Dalley JW, Theobald DE, Bouger P, Chudasama Y, Cardinal RN, Robbins TW (2004) Cortical cholinergic function and deficits in visual attentional performance in rats following $192 \mathrm{IgG}$-saporin-induced lesions of the medial prefrontal cortex. Cereb Cortex 14:922-932.

De Rosa E, Hasselmo ME (2000) Muscarinic cholinergic neuromodulation reduces proactive interference between stored odor memories during associative learning in rats. Behav Neurosci 114:32-41.

De Rosa E, Hasselmo ME, Baxter MG (2001) Contribution of the cholinergic basal forebrain to proactive interference from stored odor memories during associative learning in rats. Behav Neurosci 115:314-327.

Donner TH, Kettermann A, Diesch E, Ostendorf F, Villringer A, Brandt SA (2002) Visual feature and conjunction searches of equal difficulty engage only partially overlapping frontoparietal networks. Neuroimage $15: 16-25$

Duncan J, Humphreys GW (1989) A resemblance theory of visual search. Psychol Rev 96:433-458.

Esterman M, Verstynen T, Robertson LC (2007) Attenuating illusory binding with TMS of the right parietal cortex. Neuroimage 35:1247-1255.

Everitt BJ, Robbins TW (1997) Central cholinergic systems and cognition. Annu Rev Psychol 48:649-684.

Foldi NS, White RE, Schaefer LA (2005) Detecting effects of donepezil on 
visual selective attention using signal detection parameters in Alzheimer's disease. Int J Geriatr Psychiatry 20:485-488.

Foster JK, Behrmann M, Stuss DT (1999) Visual attention deficits in Alzheimer's disease: simple versus conjoined feature search. Neuropsychology 13:223-245.

Friedman-Hill SR, Robertson LC, Treisman AM (1995) Parietal contributions to visual feature binding: evidence from a patient with bilateral lesions. Science 269:853-855.

Furey ML, Pietrini P, Haxby JV (2000) Cholinergic enhancement and increased selectivity of perceptual processing during working memory. Science 290:2315-2319.

Hasselmo ME, McGaughy J (2004) High acetylcholine levels set circuit dynamics for attention and encoding and low acetylcholine levels set dynamics for consolidation. Prog Brain Res 145:207-231.

Himmelheber AM, Sarter M, Bruno JP (2000) Increases in cortical acetylcholine release during sustained attention performance in rats. Brain Res Cogn Brain Res 9:313-325.

Himmelheber AM, Sarter M, Bruno JP (2001) The effects of manipulations of attentional demand on cortical acetylcholine release. Brain Res Cogn Brain Res 12:353-370.

Luck SJ, Ford MA (1998) One the role of selective attention in visual perception. Proc Natl Acad Sci U S A 95:825-830.

Mesulam MM, Mufson EJ, Wainer BH, Levey AI (1983) Central cholinergic pathways in the rat: an overview based on an alternative nomenclature (Ch1-Ch6). Neuroscience 10:1185-1201.

Miranda MI, Bermúdez-Rattoni F (1999) Reversible inactivation of the nucleus basalis magnocellularis induces disruption of cortical acetylcholine release and acquisition, but not retrieval, of aversive memories. Proc Natl Acad Sci U S A 96:6478-6482.

Orsetti M, Casamenti F, Pepeu G (1996) Enhanced acetylcholine release in the hippocampus and cortex during acquisition of an operant behavior. Brain Res 724:89-96.

Parikh V, Sarter M (2008) Cholinergic mediation of attention: contribu- tions of phasic and tonic increases in prefrontal cholinergic activity. Ann N Y Acad Sci 1129:225-235.

Parikh V, Kozak R, MartinezV, Sarter M (2007) Prefrontal acetylcholine release controls cue detection on multiple timescales. Neuron 56:141-154.

Paxinos G, Watson C (1998) The rat brain in stereotaxic coordinates, Ed 4. San Diego: Academic.

Paxinos G, Watson C (2007) The rat brain in stereotaxic coordinates, Ed 6. Burlington, MA: Academic.

Pepeu G, Giovannini MG (2004) Changes in acetylcholine extracellular levels during cognitive processes. Learn Mem 11:21-27.

Reynolds JH, Desimone R (1999) The role of neural mechanisms of attention in solving the binding problem. Neuron 24:19-29, 111-125.

Robertson LC (2003) Binding, spatial attention and perceptual awareness. Nat Rev Neurosci 4:93-102.

Safer DJ, Allen RP (1971) The central effects of scopolamine in man. Biol Psychiatry 3:347-355.

Sarter M, Hasselmo ME, Bruno JP, Givens B (2005) Unraveling the attentional functions of cortical cholinergic inputs: interactions between signal-driven and cognitive modulation of signal detection. Brain Res Brain Res Rev 48:98-111.

Tales A, Butler SR, Fossey J, Gilchrist ID, Jones RW, Troscianko T (2002) Visual search in Alzheimer's disease: a deficiency in processing conjunctions of features. Neuropsychologia 40:1849-1857.

Treisman AM, Gelade G (1980) A feature-integration theory of attention. Cogn Psychol 12:97-136.

von Grünau M, Dubé S, Galera C (1994) Local and global factors of similarity in visual search. Percept Psychophys 55:575-592.

Vuckovich JA, Semel ME, Baxter MG (2004) Extensive lesions of cholinergic basal forebrain neurons do not impair spatial working memory. Learn Mem 11:87-94.

White KG, Ruske AC (2002) Memory deficits in Alzheimer's disease: the encoding hypothesis and cholinergic function. Psychon Bull Rev 9:426437. 